\title{
Positioning Control and Operational Evaluation of A One Mass Rotary System using NCTF Controller
}

\author{
Rozilawati Mohd Nor ${ }^{1}$, Sahazati Md Rozali ${ }^{1}$, Chong Shin Horng ${ }^{2}$ \\ Centre for Robotics and Industrial Automation (CeRIA) \\ ${ }^{I}$ Faculty of Electrical and Electronic Engineering Technology \\ ${ }^{2}$ Faculty of Electrical Engineering \\ Universiti Teknikal Malaysia Melaka (UTeM), Hang Tuah Jaya, 76100 Durian Tunggal, Melaka, Malaysia \\ *Corresponding author E-mail: rozilawati@utem.edu.my
}

\begin{abstract}
A practical control scheme is proposed for a one mass rotary system. It was written to demonstrate the controller performance towards positioning and tracking control. For this system, the Nominal Characteristic Trajectory Following (NCTF) controller is proposed and improved. The objective of NCTF controller is to make the object motion to follow the NCT and ends at it origin. Generally, the NCTF controller consists of a Nominal Characteristic Trajectory (NCT) obtained from open loop response and Proportional Integral (PI) compensator. The CM-NCTF controller is proposed for evaluating the motion performance and compare with the conventional NCTF controller. For positioning control, both NCTF controllers demonstrate almost identical positioning performance. However, for tracking control, CM-NCTF controller demonstrates better tracking performance than the conventional NCTF controller with the smallest motion error presented. Besides, the robustness of the CM-NCTF controller to the variation load is examined.
\end{abstract}

Keywords: rotary system; Conventional NCTF controller; CM-NCTF controller.

\section{Introduction}

Positioning system development has raised the attention of researchers and industry developers, especially for those who seek for automation development such as robotic field, machine tools, precision control and manufacturing system. An intended to have a precise and high speed positioning performance in order to maintain their product quality and quantity is very important for industry. Hence, to produce a promising positioning system, there are few things can be done such as improve the machine mechanism, use an advanced sensor or design a controller. All three features are important because each of them has significant influence towards the system. For some reason, the use of an advanced sensor or improve the machine mechanism may not suitable because it requires high cost and maintenances.

As an alternative solution, a controller will be proposed to demonstrate high accuracy, fast response, high speed and robust to uncertainties, parameter variations and disturbance. Positioning system performance usually affected by nonlinear characteristics such as actuator saturation, friction and also influences of disturbance or uncertainties. The saturation that produced by a system actuator may cause the slow system performance and affecting the system stability, while too much friction may cause too large steady state error and limit output cycles near the reference input. Hence, the designed controller must able to consider all mentioned issues to possess a promising control performance.

For a practical used in industry, classic controller has been widely use. It is because classic controller has a simple structure, easy to design, easy to understand for those who are not familiar with control field and involves low cost to design. But, this type of controller might not be sufficient for the system which has high requirement and high non-linearity. Hence, to improve this classic controller performance, some researcher comes out with an idea to hybrid the system with other type of controller such as Sliding Mode Control (SMC) and Disturbance Observer (DOB) [1]. It shows that by using by using PID Sliding Mode Control based on disturbance observer, the chattering problem was improving the dynamic and static quality of servo system. But, it only simulates on linear system and not discussing the disturbance rejection performance due to parameter variations which may happened if it tested on real time system.

The classical controller sometime has met it limitation when the system required high robustness characteristic and high positioning performance. So, there is also another approach done to produce a good precision positioning system. As an example, for high positioning performance, a robust digital controller has been designed which consist four element [2]. There is friction compensator, disturbance observer, feedback controller and also feed forward controller. The controller performance was compared with another digital tracking controller with friction compensation. The suggested controller has yield small error compared to the other controllers and can removed the disturbance and uncertainties, but it has a complexes design procedure and the system parameter variation must be known to design it.

Basically, a characterization of external friction disturbances is beneficial for servo control applications where high precision positioning is crucial [3]. For that reason, the implementation of disturbance observer was popular among researchers because it can reduce the effect of disturbance into the system. Other than above controller, a disturbance observer [4-7] approach also has been study well through many research and case study. The development of disturbance observer in control field has been formulat- 
ed in many ways either in the transfer function domain or in the state space domain.

Despite the entire controllers that have been suggested before by other researchers, most of them require exact and accurate model parameters which sometimes troublesome the researcher in the controller design procedures. Hence, the Nominal Characteristic Trajectory Following (NCTF) controller is proposed for this research. Study on NCTF Controller was done towards various type of system such as ball screw mechanism [8-9], one mass system [10], two mass system [11-12], non-contact mechanism [13] and many else.

All the system listed above required a precise positioning performance and robust to parameters variation. For a conventional NCTF Controller, NCT was constructed with PI compensator. The NCTF Controller performance is observed through experiments. It shows that it has an effective positioning performance and robust to inertia and parameters variation than a classic PID controller [10]. Same approach was done to non-contact mechanism and it also shows high positioning and tracking performance. In this paper, the NCTF Controller structured was improved to get a better positioning performance and it is known as Continuous Motion-Nominal Characteristic Trajectory Following (CM-NCTF) Controller. Compared to conventional NCTF Controller, CMNCTF Controller helps to speed up mechanism movements.

On the other hands, further study was done to reduce the overshoot and errors obtain by improving the CM-NCTF Controller. The controller was improved to Acceleration ReferenceContinuous Motion NCTF (AR-CM NCTF) Controller [14-15]. The proposed controller was examined by using mechanism with friction, which it has an ability to reduce error and overshoot of the system. The AR-CM NCTF Controller has showed a better positioning and tracking performances compared to Proportional Derivative with Disturbance Observer (PDDO) Controller. It is also having high robustness when the mechanism mass was increased.

However, sometimes the effect of actuator are not successfully compensate due to integrator windup happened to the plant [16] Large position reference that cause this matter happen and to solve the problem the compensator design for NCTF Controller was add up with the integrator windup scheme. It shows that by using PI compensator with anti-windup was more robust to inertia variation compared to conventional PI Compensator [16].

From previous study, it shows that NCTF Controller have a superior positioning and tracking performance, increase the system characteristic and also robust to inertia, friction and multi-mass application. The controller performance towards nonlinear system is very wondrous and it has a suitable characteristic to be applied for a one mass rotary system. This system is a non-linear system with a classic dynamic problems and it was always used as a benchmark for testing control theory and algorithm.

In conclusion, since the precision positioning system play important roles for industrial purposed. This research was focused on designing robust controller for one mass rotary system in order to fulfil the desired output. The CM-NCTF controller is used as a main controller to control the system positioning performance then compared with the conventional NCTF controller. Different on performance was analyzed and at the same time solve the control problem, which involving positioning system.

\section{Controller Design}

\subsection{NCTF Controller Structure}

The NCTF Controller is proposed since 2002. The controller comprises of two important elements which are nominal characteristic trajectory (NCT) and a PI compensator. The NCT is constructed on the phase plane using velocity and displacement result of an open loop experiment. While, the PI compensator will control the object motion to reach and follow the constructed NCT.
The reaching phase is occurred when the object motion completely reaches the NCT by reducing the difference between actual error rate and NCT error rate control by PI compensator. For the following phase, after the object motion reach NCT, it will then control by PI compensator to follow NCT and ends at it origin. As for the controller structure for both the Conventional NCTF and CM-NCTF controller can be seen in Figure 2 and 3. Basically, both NCTF controller have almost identical structure and control laws. However, the CM-NCTF controller is improved to rapid up the object motion and reduce the tracking motion errors.

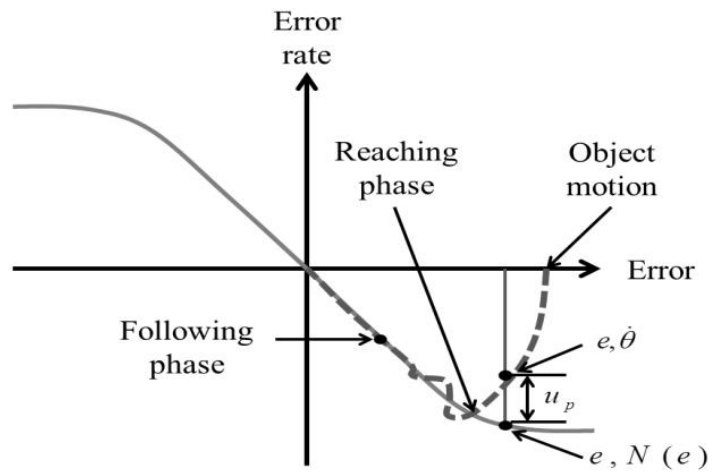

Fig. 1: NCT and object motion

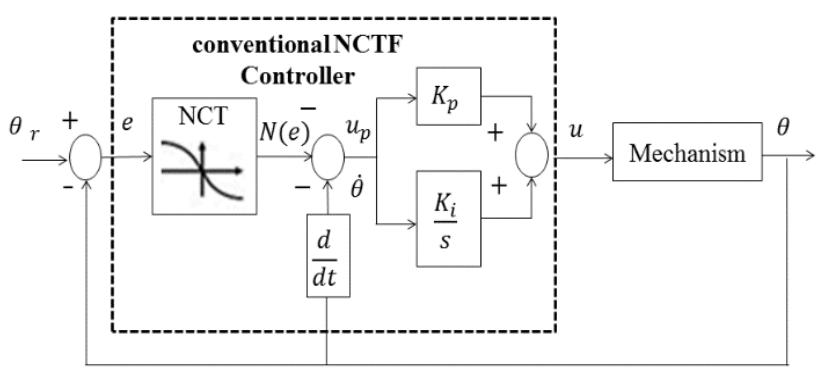

Fig 2: Conventional NCTF controller structure

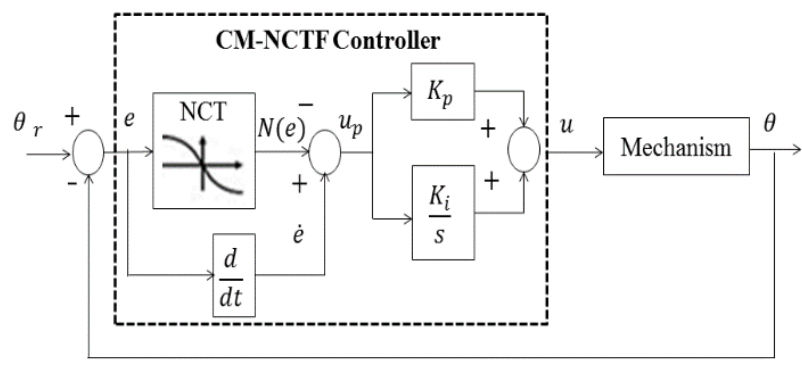

Fig. 3: CM-NCTF controller structure

The control law for both NCTF controller is derives in (1)-(3). It shows the different between actual and virtual error rate for the conventional and CM- NCTF controller. The virtual error rate is obtained from constructed NCT.

$U(s)=\left(K_{p}+\frac{K_{i}}{s}\right) U_{p}(s)$

Error rate for the conventional NCTF controller

$U_{p}(s)=\dot{E}(s)_{\text {virtual }}-\frac{d(\theta(s))}{d t}$

Error rate for the CM-NCTF controller

$U_{p}(s)=\dot{E}(s)_{\text {virtual }}-\frac{d(E(s))}{d t}$

From the equation, the CM-NCTF controller is improved by reducing the tracking motion errors by differentiate directly from 
actual errors instead of using the actual displacement of the system.

\subsection{NCTF Controller Design}

The design procedure of the CM-NCTF controller is similar to the conventional NCTF controller as shown as below:

\subsubsection{System Open Loop Response}

The system is driven by stepwise input and the displacement and velocity result is measured The input amplitude is designed to not exceed the rated input of the system to avoid the system from damaged. The selected input must obtain fast and smooth response. The specification of selected input is $10 \mathrm{~V}$ in amplitude, and sampling time 0.002 second. Figure 4 shows the open loop response result.

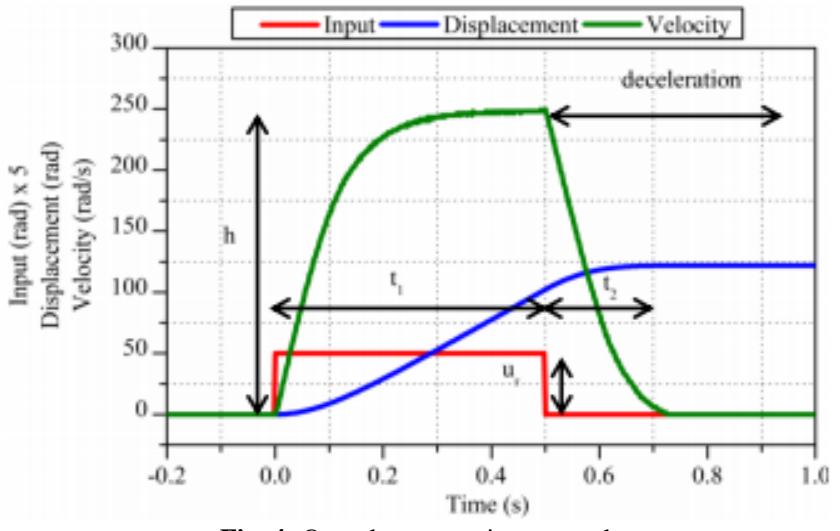

Fig. 4: Open loop experiment result

\subsubsection{NCT Construction}

The displacement and velocity results obtained from open loop experiment is used to construct the NCT. It is constructed by using only deceleration phase of displacement and velocity. Figure 4 shows the constructed-NCT with the inclination near origin of is $\beta$ $=439 \mathrm{~s}^{-1} . \beta$ is denoted as the inclination of the constructed NCT.

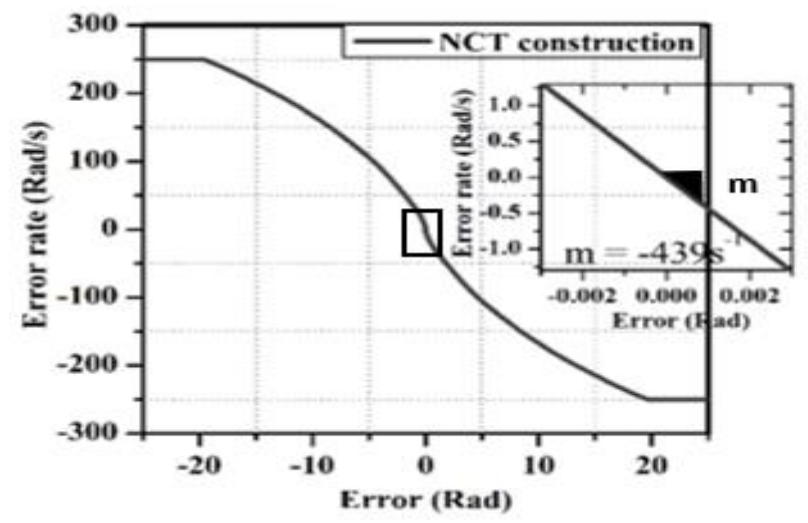

Fig. 5: Construct-NCT

\subsubsection{PI Compensator Designs}

The PI compensator is designed by using stability limit of the system. The first step is to increase the proportional gain to get the sufficient ultimate proportional gain, $u_{p}$. The sufficient $u_{p}$ is obtained when sustain periodic output yield during steady state condition, which will be use to derived the practical stability limit. Then, the simplified equation for $K_{p}$ and $K_{i}$ for the compensator is obtained as shown in (4) and (5).

$\mathrm{K}_{\mathrm{p}}=\frac{2 \xi \omega_{n} u_{r}}{m h}$
$K_{i}=\frac{\omega_{n}^{2} u_{r}}{m h}$

The suitable value of $\xi$ and $\omega_{n}$ is obtained from stability limit the maximize the performance of the PI compensator as shown in Figure 6, since NCTF controller does not require the exact parameter. Hence the parameter value of the compensator is derived from $h$ and $B$ parameter obtain from the constructed-NCT. The object is assuming to followed $2^{\text {nd }}$ order system by neglected the nonlinear characteristic as shown in (2).

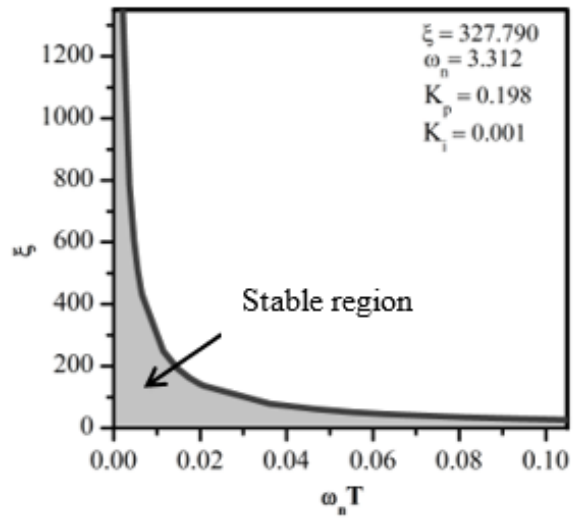

Fig. 6: Practical stability graph

\section{Methodology}

The one mass rotary system was used as the test bed to examine the effectiveness of the NCTF controller for positioning control. Figure 6 shows physical structure of a one mass rotary system. The system is driven by DC servo motor and an incremental rotary encoder with 500 counts per resolutions. The rated voltage for the DC servo motor is $\pm 10 \mathrm{~V}$ and for experiment, the sampling time used is $0.002 \mathrm{~s}$.

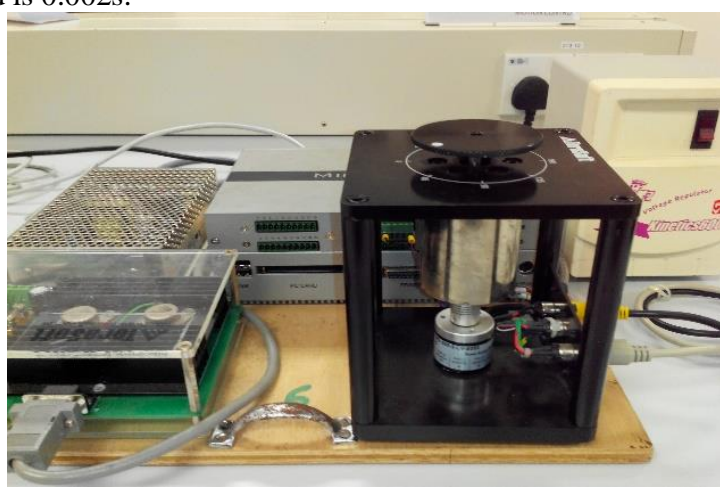

Fig. 7: A one mass rotary system

Table 1: Parameter of a one mass rotary system

\begin{tabular}{|c|c|}
\hline Armature inductance, $L_{a}$ & $4.70 \mathrm{mH}$ \\
\hline Armature resistance, $R_{a}$ & $2.56 \Omega$ \\
\hline Back EMF constant, $K_{b}$ & $1.83 \times 10^{-2} \mathrm{NmA}^{-1}$ \\
\hline Torque constant, $K_{t}$ & $1.83 \times 10^{-2} \mathrm{NmA}^{-1}$ \\
\hline Viscous friction coefficient, $B$ & $9.14 \times 10^{-5} \mathrm{Nmsrad}^{-1}$ \\
\hline Motor inertia, $J_{m}$ & $7.20 \times 10^{-5} \mathrm{kgm}^{2}$ \\
\hline Load mass, $m_{1}$ & $0.075 \mathrm{~kg}$ \\
\hline
\end{tabular}

\section{Positioning Performance}

\subsection{Positioning Control Performance}

The experiment was done using three different positions which are 1 radian, 2 radian and 4 radian input. Figure 7-9 show the positioning control experiment result for different input displacement. From the result, it is demonstrating that the Conventional NCTF controller has almost identical positioning response with CMNCTF controller. As the step input increase, both Conventional 
NCTF and CM-NCTF controller, no or slightly overshoot produce when small step input nor large step input applied to the system.

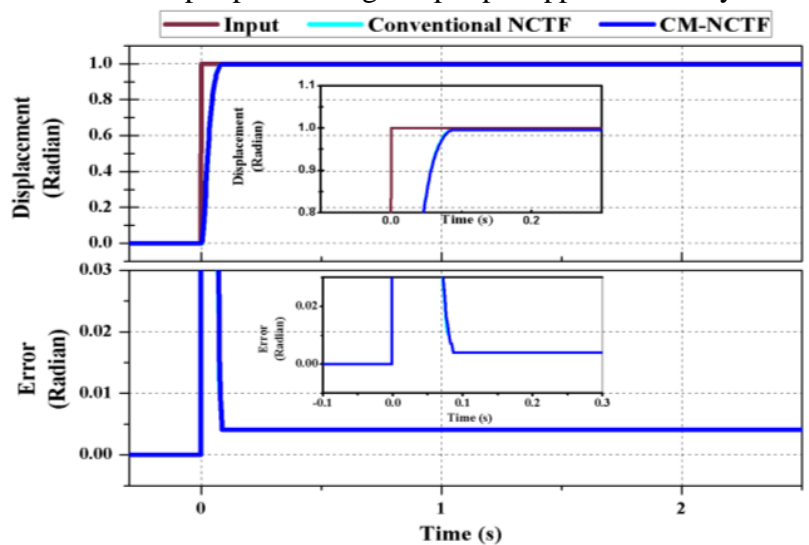

Fig. 7: Positioning 1 radian input
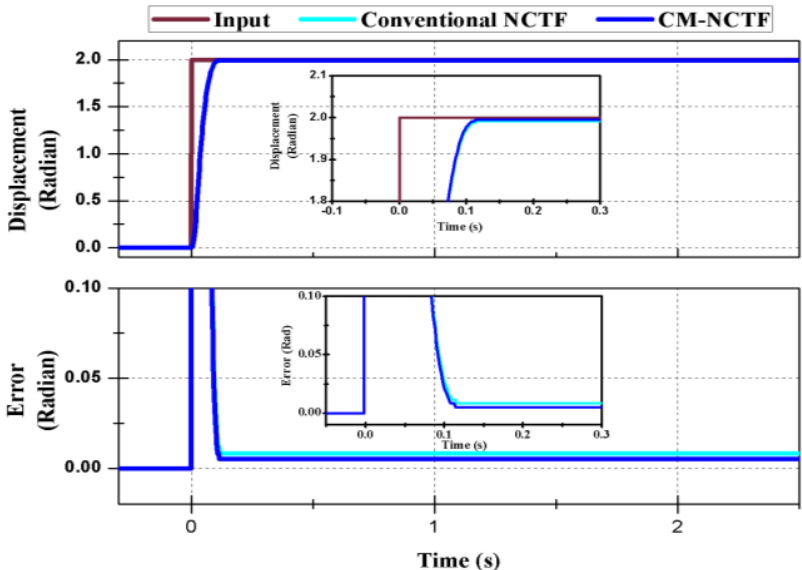

Fig. 8: Positioning 2 radian input
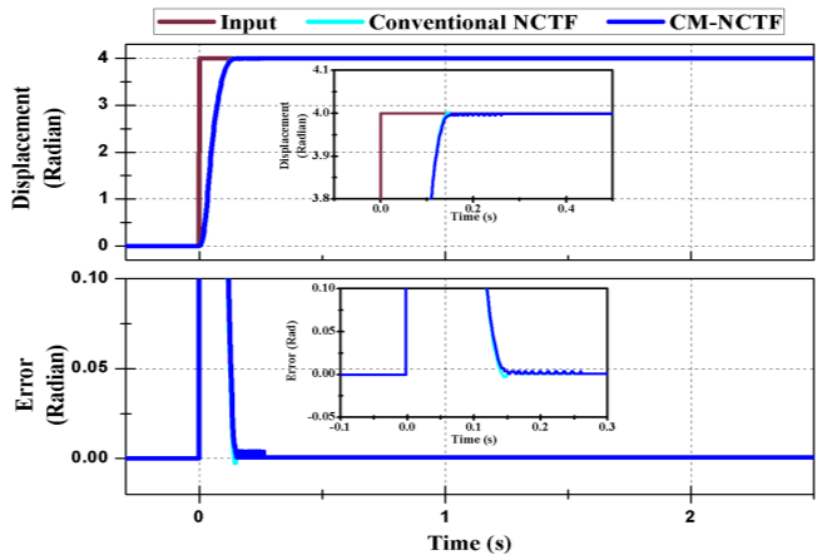

Fig. 9: Positioning 4 radian input

To see the robustness of the system, 10 times repeatability was done for positioning control experiment. Table 2 shows the positioning control performance for 10 times repeatability. It can be seen clearly that the result obtained for the conventionanl NCTF and CM-NCTF controller is almost identical.

\begin{tabular}{|c|c|c|c|c|}
\hline Controller & $\begin{array}{l}\text { Displacement } \\
\text { (Radian) }\end{array}$ & $\begin{array}{l}\text { Settling } \\
\text { Time (s) }\end{array}$ & $\begin{array}{c}\text { Error } \\
\text { (Radian) } \\
\times 10^{-3}\end{array}$ & $\begin{array}{c}\text { Overshoot } \\
(\%)\end{array}$ \\
\hline \multirow{3}{*}{$\begin{array}{l}\text { Conventional } \\
\text { NCTF }\end{array}$} & 1 & 1.07 & 4.10 & 0 \\
\hline & 2 & 1.09 & 5.10 & 0 \\
\hline & 4 & 1.12 & 6.80 & 0 \\
\hline \multirow{3}{*}{ CM-NCTF } & 1 & 1.07 & 3.80 & 0 \\
\hline & 2 & 1.09 & 5.10 & 0 \\
\hline & 4 & 1.12 & 6.70 & 0 \\
\hline
\end{tabular}

* 10 times repeatability
Both NCTF controller demonstrate less steady state error. However, as the reference input increse, the error produce also increase. It is also shows fast rise time and zero overshoot. From here, it shows that both NCT controller can perform well for positioning performance. However, motion control cannot only relis on positioning response but tracking control performance need to be considered too.

\subsection{Tracking control performance}

For tracking control performance, different amplitude and frequency use to evaluate the controller performance. For this case, 1 and 3 radian amplitude with 0.3 and $0.7 \mathrm{~Hz}$ frequency used. Figure 10-13 show the tracking motion performance for both NCTF controller.

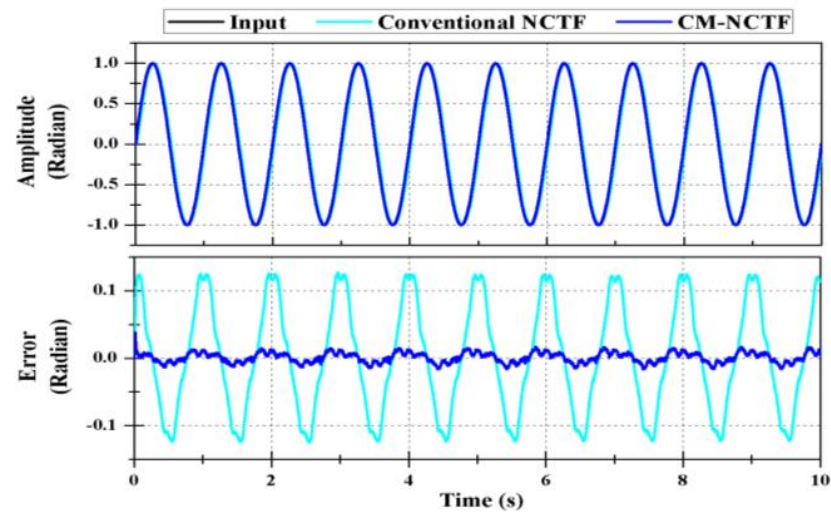

Fig. 10: Tracking with frequency $0.3 \mathrm{~Hz}$, amplitude 1 radian
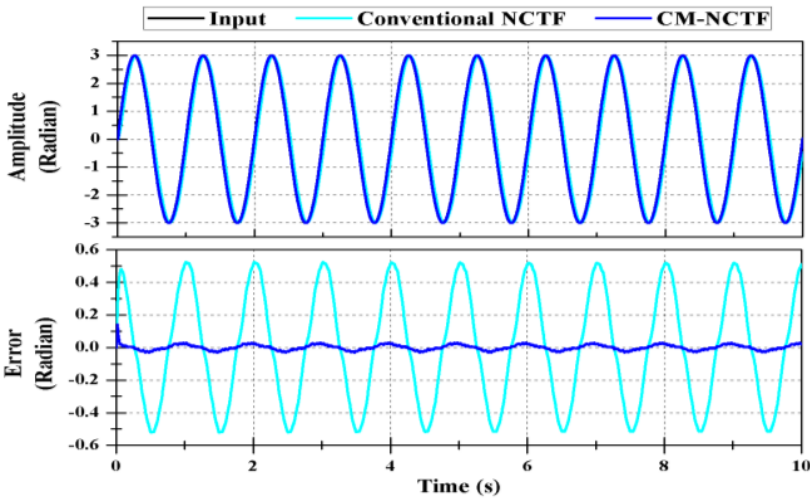

Fig. 11: Tracking with frequency $0.3 \mathrm{~Hz}$, amplitude 3 radian

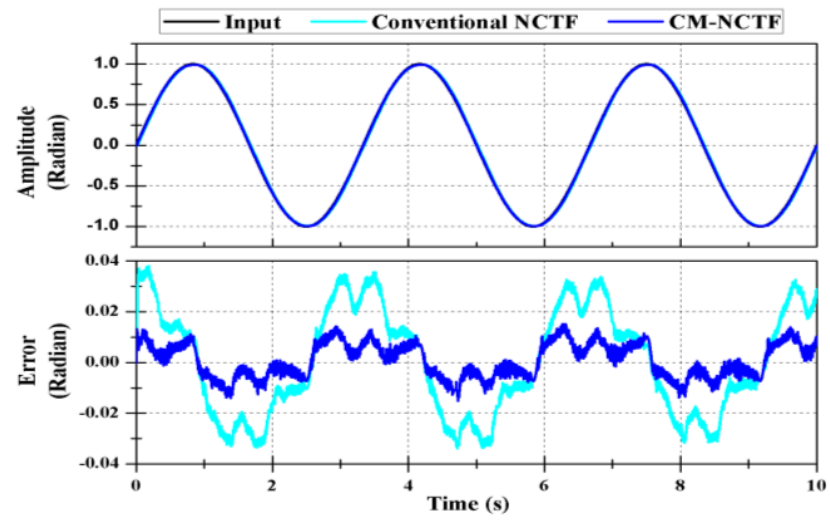

Fig. 12: Tracking with frequency $1 \mathrm{~Hz}$, amplitude 1 radian

From tracking result, it shows that with different amplitude and frequency, less error yield by CM-NCTF controller. The error produce is as shown in Table 3 with 10 times repeatability. 


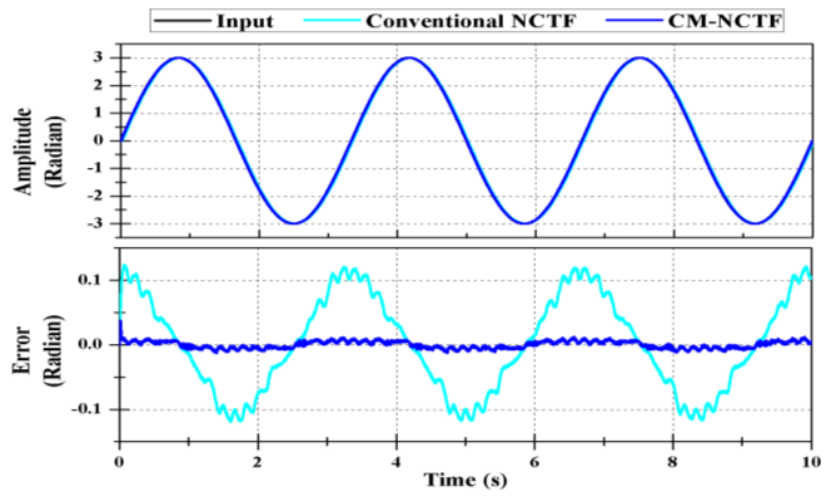

Fig. 13: Tracking with frequency $1 \mathrm{~Hz}$, amplitude 3 radian

\begin{tabular}{|c|c|c|c|}
\multicolumn{4}{c}{ Table 3: Tracking performance comparison } \\
\hline \multirow{4}{*}{ Controller } & $\begin{array}{c}\text { Amplitude } \\
\text { (Radian) }\end{array}$ & $\begin{array}{c}\text { Frequency } \\
(\mathrm{Hz})\end{array}$ & $\begin{array}{c}\text { Error } \\
\text { (Radian) } \times 10^{-2}\end{array}$ \\
\hline \multirow{4}{*}{$\begin{array}{c}\text { Conventional } \\
\text { NCTF }\end{array}$} & 1 & 0.3 & 7.17 \\
& 2 & 1.0 & 25.17 \\
\cline { 2 - 4 } & & 0.3 & 13.24 \\
& 3 & 1.0 & 64.43 \\
\hline \multirow{4}{*}{ CM-NCTF } & 1 & 0.3 & 23.47 \\
& & 1.0 & 103.39 \\
\cline { 2 - 4 } & 2 & 1.0 & 4.03 \\
& & 0.3 & 5.42 \\
\cline { 2 - 4 } & 3 & 1.0 & 4.38 \\
& & 0.3 & 10.01 \\
\hline
\end{tabular}

* 10 times repeatability

For 1 radian amplitude with $0.3 \mathrm{~Hz}$ frequency, conventional NCTF controller produce about 3\% larger error than CM-NCTF controller. But, as the ampliture and frequency increase, the error produce by the conventional NCTF is increase almost $40 \%$ from the previous result. From overall tracking result, it is clearly shows that CM_NCTF controller deonstrate better tracking performance compared to the conventional NCTF controller.

\section{Robustness Evaluation}

To evaluate the robustness of the system, experiment was done by using different load to analyze the motion performance. Including the actual load for the system, two other different mass has been applied to the system which is 120 and $208 \mathrm{~g}$ to examine the system performance towards mass changing, different amplitude and frequency use for positioning and tracking experiment. System performance result with 10 times repeatability is as shown in Table 4. From observation, even with different load applied to the system, both NCTF still demonstrate almost identical positioning response but when it comes to the tracking performance, CMNCTF controller demonstrate a better tracking performance.

Table 4: Performance comparison

\begin{tabular}{|c|c|c|c|c|}
\hline Controller & $\begin{array}{c}\text { Input } \\
\text { (Radian) } \\
\text { Frequency } \\
0.7 \mathrm{~Hz}\end{array}$ & Load & $\begin{array}{c}\text { Positioning } \\
\text { Error } \\
\text { (Radian) } \\
\times 10^{-3}\end{array}$ & $\begin{array}{c}\text { Tracking } \\
\text { Error } \\
\text { (Radian) }\end{array}$ \\
\hline \multirow{3}{*}{ Conventional } & 1 & $M_{1}$ & 4.10 & 16.74 \\
NCTF & & $M_{2}$ & 2.20 & 15.69 \\
\cline { 2 - 5 } & \multirow{2}{*}{2} & $M_{3}$ & 2.00 & 18.08 \\
\hline \multirow{3}{*}{ CM-NCTF } & & $M_{1}$ & 5.10 & 39.87 \\
& & $M_{2}$ & 1.20 & 39.91 \\
& & $M_{3}$ & 1.10 & 41.14 \\
\cline { 2 - 5 } & & $M_{2}$ & 3.80 & 4.60 \\
& \multirow{2}{*}{2} & $M_{3}$ & 2.20 & 4.41 \\
& & $M_{1}$ & 5.10 & 6.06 \\
\hline
\end{tabular}

* 10 times repeatability

\section{Conclusion}

For the conclusion, positioning and tracking control experiment done using CM-NCTF controller demonstrates better response of all. Even though Conventional NCTF controller demonstrate almost identical positioning performance, but it has a bad tracking result when larger input and higher frequency applied. When different mass applied to the mechanism, CM-NCTF also produce the smoother response of all and it prove that CM-NCTF controller is robust to parameter variation either frequency, position or mass changing.

\section{Acknowledgement}

This work was supported in part by UTeM under Grant Nos. PJP/2018/FTK(17C)/S01643.

\section{References}

[1] H. Liu, Y. J. Wu, and J. Zhang, "PID sliding mode control based on disturbance observer," Proceedings of the Chinese Control and Decision Conference, 2011, pp. 1278-1281.

[2] H. S. Lee and M. Tomizuka, "Robust motion controller design for high-accuracy positioning systems," IEEE Trans. Ind. Electron., 43(1), 48-55, 1996.

[3] W. B. Dunbar, R. A. de Callafon, and J. B. Kosmatka, "Coulomb and viscous friction fault detection with application to a pneumatic actuator," Proceedings of the IEEE/ASME International Conference on Advanced Intelligent Mechatronics, 2001, pp. 12391244.

[4] K.-Y. Chen, "Robust optimal adaptive sliding mode control with the disturbance observer for a manipulator robot system," Int. J. Control. Autom. Syst., 16(4), 1-15, 2018.

[5] Z. Chu, D. Zhu, and B. Sun, "Adaptive trajectory tracking control for remotely operated vehicles based on disturbance observer," Proceedings of the International Conference on Intelligent Robotics and Applications, 2018, pp. 137-146.

[6] X. Wang, X. Su, and L. Sun, "Disturbance observer-based singularity-free trajectory tracking control of uncertain quadrotors with input saturation," Proceedings of the Chinese Control and Decision Conference, 2018, pp. 5780-5785.

[7] W.-H. Chen, "Disturbance observer based control for nonlinear systems," IEEE/ASME Transactions on Mechatronics, 9(4), 706710, 2004.

[8] G. J. Maeda and K. Sato, "Practical control method for ultraprecision positioning using a ballscrew mechanism," Precis. Eng., 32(4), 309-318, 2008.

[9] K. Sato and G. J. Maeda, "A practical control method for precision motion-Improvement of NCTF control method for continuous motion control," Precis. Eng., 33(2), 175-186, 2009.

[10] K. Sato, A. Shimokohbe, and Wahyudi, "Characteristics of practical control for point-to-point (PTP) positioning systems," Precis. Eng., 27(2), 157-169, 2003.

[11] F. My, Wahyudi, R. Akmeliawati, and A. A. Wijaya, "Improved NCTF control method for a two mass point-to-point positioning system," Proceedings of the International Conference on Intelligent and Advanced Systems, 2010, pp. 1-6.

[12] F. My, R. Akmeliawati, and A. Wijaya, NCTF control method of two mass system for PTP positioning. Universiti Malaysia Perlis, 2009.

[13] S. Chong and K. Sato, "Practical controller for precision positioning system - A new design approach and application to mechanism with friction," Proceedings of the Am. Soc. Precis. Eng. Meet., 2010, pp. 1-6.

[14] S. H. Chong and K. Sato, "AR-CM NCTF control for precision positioning systems - Concept and results," Proceedings of the Sixth Asia Modelling Symposium, 2012, pp. 156-160.

[15] S. H. Chong and K. Sato, "Practical and robust control for precision motion: AR-CM NCTF control of a linear motion mechanism with friction characteristics," IET Control Theory Appl., 9(5), 745-754, 2015.

[16] L. Burlion and H. de Plinval, "Vision based anti-windup design with application to the landing of an airliner," IFAC-PapersOnLine, 50(1), 10482-10487, 2017. 